\title{
A NOVEL ONE-SHOT ANASTOMOTIC STAPLER PROTOTYPE FOR CORONARY BYPASS GRAFTING ON THE BEATING HEART: FEASIBILITY IN THE PIG
}

Robin H. Heijmen, MDa

Peter Hinchliffe ${ }^{b}$

Cornelius Borst, $\mathrm{MD}, \mathrm{PhD}^{\mathrm{a}}$

Cees W. J. Verlaan ${ }^{\mathrm{a}}$

Chantal M. Mouës, MDa

Yvonne J. M. van der Helma

Scott Manzo ${ }^{\mathrm{b}}$

Erik W. L. Jansen, MD, PhD ${ }^{\mathrm{a}}$

Paul F. Gründeman, MD, $\mathrm{PhD}^{\mathrm{a}}$
Objective: The nonpenetrating, arcuate-legged clip has proved its ability to provide a high-quality microvascular anastomosis. This study assessed the feasibility of constructing a coronary end-to-side anastomosis on the beating heart with a novel mechanical, sutureless anastomotic device that applies 12 circumferential clips simultaneously. Methods: In 14 consecutive pigs $(70-90 \mathrm{~kg})$, the left internal thoracic artery (diameter, $3 \mathrm{~mm}$ ) was grafted to the left anterior descending coronary artery (diameter, $3 \mathrm{~mm}$ ) by means of a one-shot anastomotic stapler prototype. Endothelial denudation, medial necrosis, and intimal hyperplasia were analyzed quantitatively and compared with those seen in conventionally sutured anastomoses $(n=4)$. Results: In 8 of 14 anastomoses, the one-shot anastomotic stapler successfully applied all 12 clips circumferentially across the everted arteriotomy edges. In the remaining, either $1(n=4)$ or 3 and 4 adjoining malaligned clips had to be replaced manually with a single-clip applicator. Coronary occlusion was limited to approximately 3 minutes. At follow-up, all anastomoses were patent angiographically. At 2 days, in 2 of 7 cases, a local coronary dissection was observed, and there was a considerable loss of endothelial cells and medial damage. At 28 days, however, minimal intimal hyperplasia was seen at the anastomotic lining, although more pronounced when compared with conventionally sutured anastomoses. Conclusions: The one-shot anastomotic stapler prototype enabled short-occlusive (3 minutes), sutureless end-to-side grafting on the beating porcine heart. In spite of early endothelial and medial damage and 2 local dissections, all anastomoses remained patent with minimal intimal hyperplasia at 4 weeks. (J Thorac Cardiovasc Surg 1999;117:117-25)
$\mathrm{R}$ ecent progress in coronary artery bypass grafting $(\mathrm{CABG})$ without cardiopulmonary bypass ${ }^{1-4}$ and initiatives toward reduced surgical access, ${ }^{5-8}$ or even a totally thoracoscopic approach, ${ }^{9-10}$ warrant an intensified search for facilitated end-to-side anastomosis techniques. ${ }^{11}$

From the Departments of Cardiology and Cardiothoracic Surgery, Heart Lung Institute, Utrecht University Hospital, ${ }^{a}$ Utrecht, The Netherlands, and United States Surgical Corporation, ${ }^{\mathrm{b}}$ Norwalk, Conn.

The study and R.H.H., C.W.J.V., and P.F.G. were supported by a grant from the Utrecht University Hospital (1995/B903).

Received for publication May 5, 1998; revisions requested June 29, 1998; revisions received July 23, 1998; accepted for publication Sept 8, 1998.

Address for reprints: Cornelius Borst, $\mathrm{MD}, \mathrm{PhD}$, Professor of Experimental Cardiology, Utrecht University Hospital (Room G02.523), PO Box 85500, 3508 GA Utrecht, The Netherlands.

Copyright (C) 1999 by Mosby, Inc.

$0022-5223 / 99 \$ 8.00+0 \quad \mathbf{1 2} / \mathbf{1} / \mathbf{9 4 2 8 1}$
To date, the coronary anastomosis is sutured under direct vision through a median sternotomy or minithoracotomy, while the coronary artery is temporarily occluded. Performing a coronary anastomosis on a beating heart, however, particularly via limited access, is technically demanding. Video-assisted, closed-chest CABG may further complicate the procedure and prolong the occlusive anastomosis time ${ }^{10}$ and the ischemic period. The potential risk of regional myocardial ischemic injury as the result of prolonged coronary occlusion may be circumvented by accelerated tissuebonding techniques. In 1992, Kirsch and colleagues ${ }^{12}$ introduced a new method for microvascular reconstruction based on the principle of flanged, intimal approximation by interrupted application of arcuate-legged, nonpenetrating clips. To date, successful clipped anastomoses have been performed clinically in both the peripheral ${ }^{13,14}$ and coronary circulation. ${ }^{15}$ Symmetrical eversion of the vessel edges and proper application of 


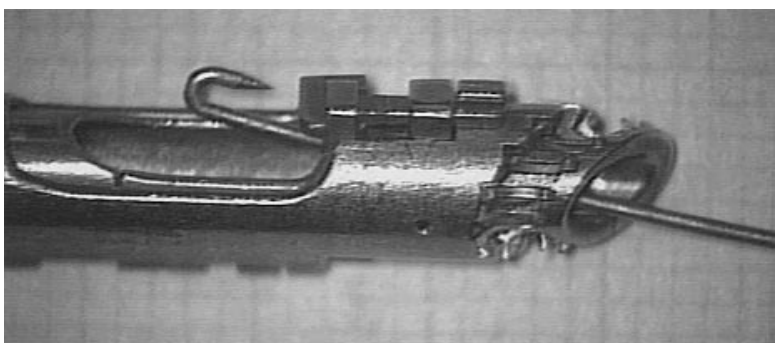

Fig 1. Close-up of the tip of the one-shot anastomotic stapler. The clip cartridge is loaded circumferentially with 12 equally spaced, medium-sized clips (VCS; USSC, Norwalk, Conn). To accommodate the distal end of the ITA graft in the clip cartridge, all loose periadventitial tissue and hemostatic clips for side branches were gently removed over a distance of approximately $3 \mathrm{~cm}$. The ITA was hooked on and subsequently pulled through the clip cartridge. The end of the graft was cut at a 45-degree angle, to conform to the angled tip of the clip cartridge. The distal end of the ITA was everted across the anvil and carefully hooked on the lower jaws of the clips. Next, the clip cartridge with the everted distal end of the ITA was attached to the firing handle. The everted and exposed intimal layer of the graft was frequently irrigated with saline solution to avoid the occurrence of dehydration.

the clips requires training, however. To facilitate eversion and to apply the clips circumferentially by a single maneuver, enabling rapid end-to-side anastomosis, a one-shot anastomotic stapler (One-Shot; US Surgical Corporation* [USSC], Norwalk, Conn) was developed.

The aim of this study was to assess the feasibility of applying the early prototype of the one-shot anastomotic stapler during left internal thoracic artery (ITA) bypass grafting to the left anterior descending coronary artery (LAD) on the beating heart in the pig and to investigate quantitatively coronary artery wall injury and healing in comparison with conventional suturing.

\section{Materials and methods}

Animals. Fourteen consecutive Dutch landrace pigs (weight, 70-90 kg) were used. One day before the operation, all pigs received acetylsalicylic acid (500 mg, orally). This was continued (160 mg/day) until they were killed. The animals received humane care in compliance with the "Guide for the Care and Use of Laboratory Animals" prepared by the Institute of Laboratory Animal Resources and published by the National Institutes of Health (NIH Publication No 86-23, revised 1985). All procedures performed in this study were

*Except for making the device available, this study was not funded by US Surgical Corporation.

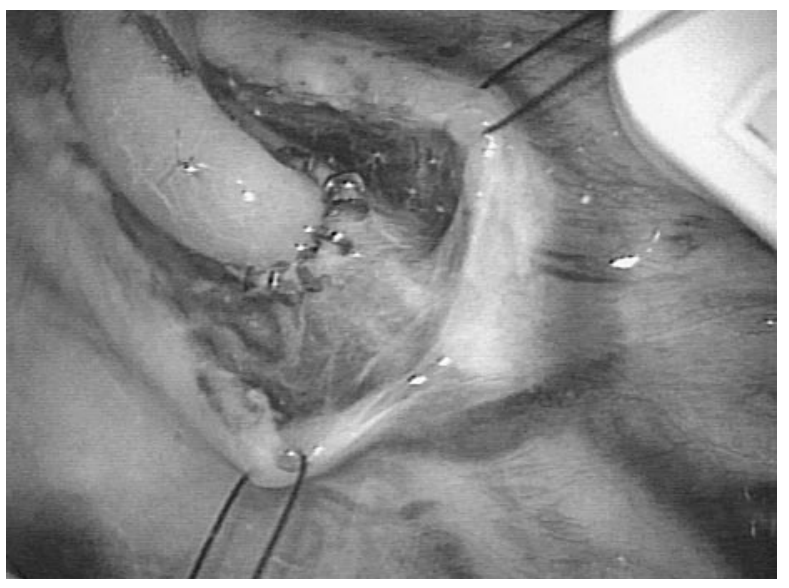

Fig 2. Clipped left ITA-LAD anastomosis on the beating procine heart, after restoration of graft blood flow. The LAD was immobilized with the "Octopus" (Octopus Tissue Stabilizer; Medtronic, Inc, Minneapolis, Minn; upper right corner). To improve visualization, 2 sutures were passed through the epicardium on either side of the LAD. Side branches located in the trimmed distal end of the ITA graft were occluded by either a hemostatic clip or suture.

approved by the Animal Experimentation Committee of the Utrecht University. Four pigs from a different series of comparable experiments served as controls. ${ }^{16}$

Study protocol. The animals were anesthetized and monitored hemodynamically as described before. ${ }^{16}$ After median sternotomy, the left ITA was harvested in a skeletonized fashion. After heparinization $(150 \mathrm{IU} / \mathrm{kg})$, the ITA was transected and flushed with papaverine-saline solution $(0.5 \%)$ to prevent spasm. After the pericardium was opened, the proximal one third of the LAD, near the first diagonal branch, was immobilized by 2 Octopus tentacles (Octopus Tissue Stabilizer; Medtronic, Inc, Minneapolis, Minn) ${ }^{8,17}$ For adequate exposure of the target area, the heart was slightly dislocated medially and hoisted ventrally, and two 4-0 polypropylene sutures were passed through the epicardium on either side of the LAD.

All 14 one-shot ITA-LAD anastomoses and the 4 conventionally sutured anastomoses were performed under the operating microscope (magnification, $\times 6$ to $\times 12$; Wild M680; Leica AG, Heerbrugg, Switzerland) by one investigator (R.H.H.) and recorded on video tapes for retrospective analysis.

Phasic and mean blood flow rate of the ITA graft were measured with a transit time flow probe (4S; Transonic, Ithaca, NY) connected to a flowmeter (T208; Transonic, Ithaca, NY) and recorded on an 8-channel recorder. The baseline mean flow rate and the coronary hyperemic response were measured as described before. ${ }^{16}$

Before the animals were killed, the anastomosis in all animals was visualized by left ITA angiography with frontal and right anterior oblique projections (C-arm BV27; Philips, Eindhoven, The Netherlands). The pigs were evaluated at 2 days $(n=7)$ and at 28 days $(n=7)$ after operation. 


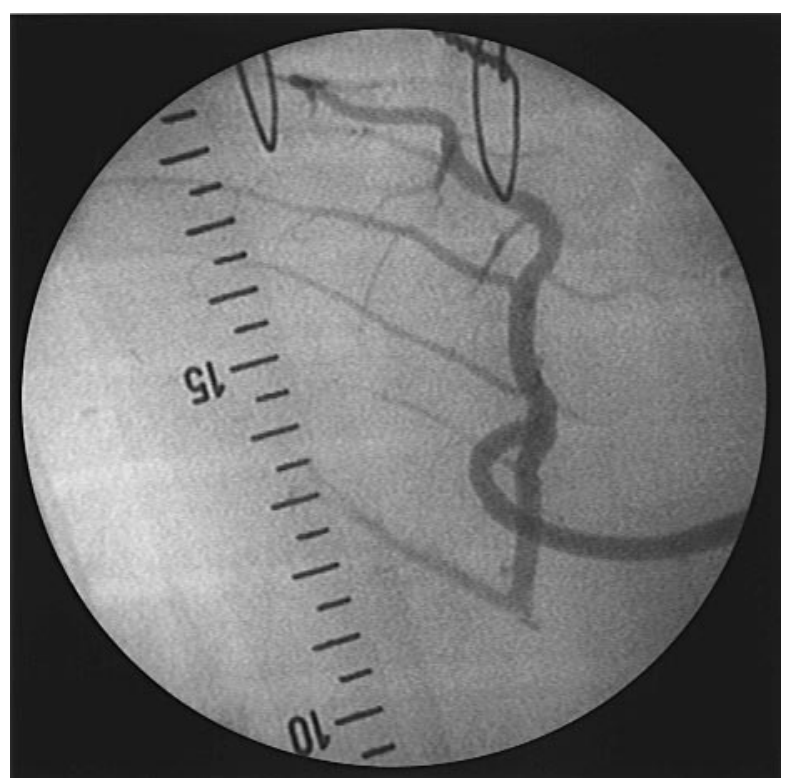

Fig 3. Representative angiogram at 28 days after the operation, revealing a patent left ITA-LAD anastomosis. The angiographically determined luminal diameter of the ITA graft and LAD at the anastomosis site was $3.1 \pm 0.4 \mathrm{~mm}$ (range, 2.3-3.7 $\mathrm{mm}$ ) and $3.0 \pm 0.5 \mathrm{~mm}$ (range, $2.3-3.7 \mathrm{~mm}$ ), respectively.

Anastomotic procedure. The one-shot anastomotic stapler consists of a clip cartridge (Fig 1), attached to a firing handle that serves for simultaneous, circumferential clip closure. The distal end of the ITA graft was pulled through the clip cartridge, everted across the anvil, and carefully hooked on the lower jaws of the clips. After proximal coronary occlusion by means of a microvascular Acland clamp (B-3V; S\&T Marketing Ltd, Neuhausen, Switzerland), a standardized arteriotomy was created with the use of an elliptic vascular punch $(2.75 \times 1.25 \mathrm{~mm}$; USSC). Next, the everted distal end of the ITA was inserted in the arteriotomy by means of a pivoting motion. After all sides were scrutinized for proper alignment, the firing handles were squeezed, closing the clips while their upper jaws grasped and subsequently everted the LAD. Gently lifting the instrument released the closed clips from their slots. Resqueezing the firing handles allowed the clip cartridge to split and enabled removal of the ITA graft from within it. After restoration of ITA graft flow, the LAD was occluded permanently proximal to the anastomosis. Side branches that happened to be located in the trimmed distal end of the ITA were occluded by either a hemostatic clip or 8-0 polypropylene suture.

Histologic analysis. To preserve anastomotic geometry, all arteries were pressure-fixed at $80 \mathrm{~mm} \mathrm{Hg}$, as described before. ${ }^{18}$ After overnight fixation in formalin $4 \%$, the clips were carefully removed with a special clip remover (USSC). The anastomotic segments were embedded in paraffin and subsequently sectioned in the transverse plane at $200-\mu \mathrm{m}$ intervals. Sections were stained with hematoxylin and eosin

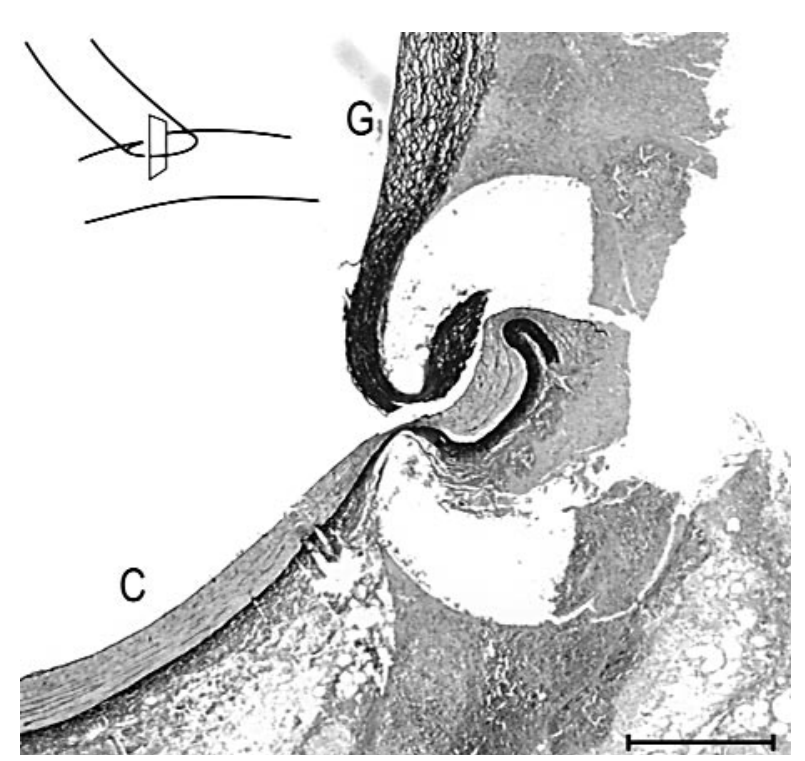

Fig 4. Transverse cross-section of the anastomotic line in the mid-segment of the anastomosis, 2 days after the operation. The blank space corresponds to the position of a clip after its removal. Note the perfect apposition of intima-to-intima. (Elastin van Gieson stain; bar, $500 \mu \mathrm{m}$.) $G$, Graft; $C$, coronary artery.

Table I. Graft blood flow and coronary hyperemic response, postoperative and at follow-up*

\begin{tabular}{|c|c|c|c|c|}
\hline & $n$ & $\begin{array}{l}\text { After the } \\
\text { operation }\end{array}$ & Follow-up & $\begin{array}{c}\mathrm{P} \\
\text { value }\end{array}$ \\
\hline \multicolumn{5}{|c|}{$\begin{array}{l}\text { Graft blood flow } \\
(\mathrm{mL} / \mathrm{min})\end{array}$} \\
\hline 2 Days & 7 & $18 \pm 6$ & $59 \pm 36$ & .02 \\
\hline 28 Days & 7 & $20 \pm 7$ & $47 \pm 15$ & .002 \\
\hline Total & 14 & $19 \pm 6$ & $53 \pm 27$ & .001 \\
\hline \multicolumn{5}{|c|}{$\begin{array}{l}\text { Coronary } \\
\text { hyperemic response }\end{array}$} \\
\hline 2 Days & 7 & $4.0 \pm 0.8$ & $3.1 \pm 0.7$ & .03 \\
\hline 28 Days & 7 & $4.0 \pm 0.7$ & $4.9 \pm 1.2$ & .23 \\
\hline Total & 14 & $4.0 \pm 0.7$ & $4.0 \pm 1.3$ & .92 \\
\hline
\end{tabular}

At follow-up, a threefold increase in baseline mean flow rate of the ITA graft was observed. The coronary hyperemic response remained higher than 3, suggesting no impediment to blood flow.

*Results are presented as the mean \pm SD of 2 determinations at an interval of 10 minutes. The coefficient of variation was $9 \% \pm 3 \%$ (range, $4 \%-17 \%$ ) $\dagger$ Postoperative vs follow-up, paired Student $t$ test (2-tailed).

and with elastin van Gieson. Light microscopy was used to identify endothelial injury, medial necrosis, and intimal hyperplasia, as described before. ${ }^{16}$

Previously performed conventionally sutured ITA-LAD anastomoses off-pump from a different series of comparable experiments ${ }^{16}$ were analyzed identically and served as controls for both endothelial injury $(n=2)$ and intimal hyperplasia $(n=2)$. 


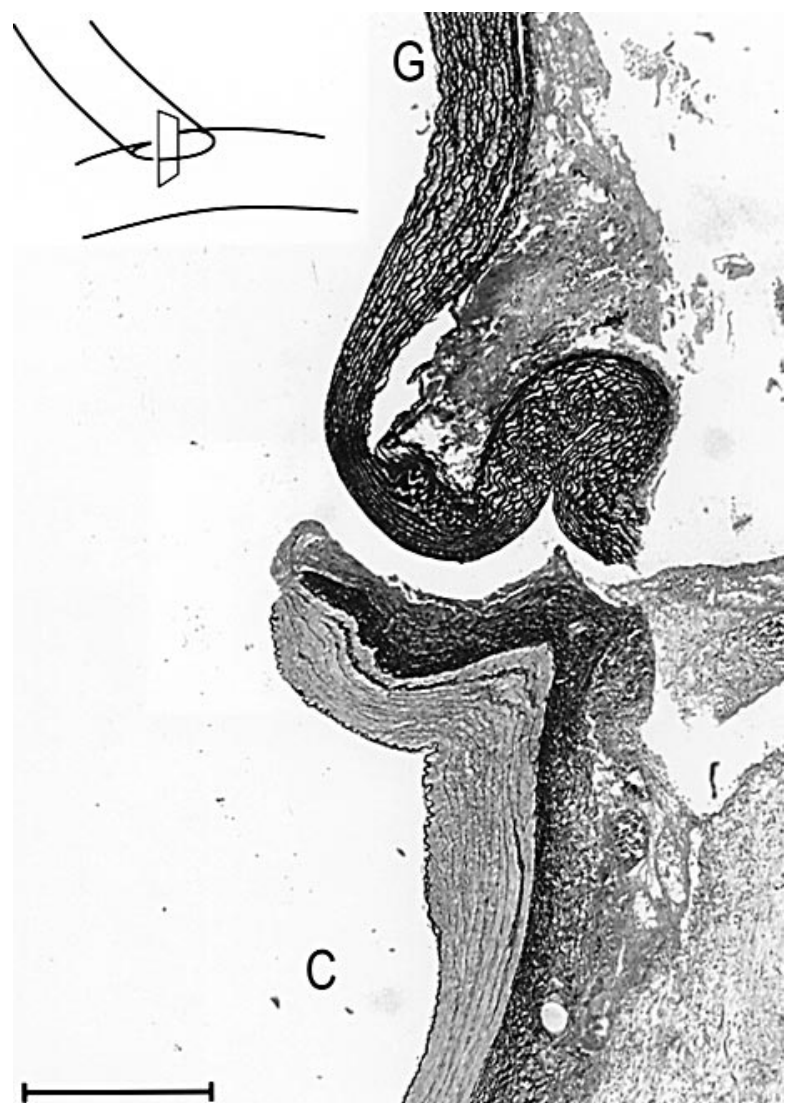

Fig 5. Transverse cross section of the anastomotic line in the mid-segment of the anastomosis, 2 days after the operation. In 5 of 14 anastomoses, the coronary arterial wall has not been everted adequately at the heel $(\mathrm{n}=3)$ or lateral wall $(\mathrm{n}$ $=2$ ) of the anastomosis. (Elastin van Gieson stain; bar, 500 $\mu \mathrm{m}$.) $G$, Graft; $C$, coronary artery.

Statistical analysis. Data are presented as mean \pm standard deviation or as median and range. The paired Student $t$ test (2tailed) was used to compare mean values.

\section{Results}

Anastomotic procedure. In 7 of 14 anastomoses, the one-shot anastomotic stapler successfully applied all 12 clips simultaneously across the everted edges of the ITA graft and LAD (Fig 2). In 4 anastomoses, one clip either had missed the LAD $(n=3)$ or was not properly closed because of premature release out of its slot. In 2 anastomoses, 3 and 4 adjoining clips at the toe and the right side of the anastomosis, respectively, missed the LAD. In one anastomosis, adequacy of insertion was misjudged. After the one-shot device had been fired, none of the circumferentially applied clips had closed across the arteriotomy edge of the LAD.
After the distal end of the ITA had been transected, everted onto a second clip cartridge, and subsequently reinserted in the arteriotomy, the second attempt of circumferential clip closure was successful. This increased coronary occlusion time from about 3 to nearly 14 minutes. In the 6 imperfect anastomoses, malaligned clips were replaced manually by a mediumsized single-clip applier (USSC), which required 98 seconds (range, 13-206 seconds). Eversion of the distal end of the ITA graft onto the lower jaws of the clips required 51 seconds (range, 20-165 seconds; outlier, 607 seconds). The outlier was caused by repetitive tearing of the arterial wall on eversion. In 13 of 14 cases, the toe of the everted ITA graft could not be secured on the clips. Alternatively, the ITA was attached to a small pin proximal to the clips, thereby covering both jaws of 2 to 3 clips at the toe completely. The time required for arteriotomy with a vascular punch was 26 seconds (range, 15-125 seconds). In 2 of 14 cases, the punched hole was incomplete and required manual trimming. Insertion of the distal end of the ITA graft in the arteriotomy together with close inspection of the arteriotomy edges required $123 \pm 40$ seconds. After the one-shot device was fired, the closed clips were released from their slots in $21 \pm 6$ seconds. The ITA graft was removed from within the clip cartridge in $9 \pm 5$ seconds. The duration of coronary occlusion during construction of the graft was 209 seconds (range, 150-373 seconds; outlier, 830 seconds). The ITA-LAD anastomosis was completed in 4 minutes (range, 3-14 minutes) and required 12 clips (range, 12-15 clips). After restoration of ITA graft flow, instant hemostasis was observed in 5 of 7 anastomoses, with successful circumferential clip closure. Two anastomoses showed initial oozing of blood between the everted edges for about 40 seconds. In 7 of 14 anastomoses, one $(n=5)$ or two side branches that were located in the trimmed distal portion of the ITA graft required closure by a hemostatic clip or suture afterwards. In 10 of 14 animals, no ventricular arrhythmias other than occasional ventricular premature beats were observed during the operative procedure. In 4 pigs, however, abrupt ventricular fibrillation occurred after a mechanical stimulus $(\mathrm{n}=3)$ and during construction of the anastomosis after only $90 \mathrm{sec}-$ onds of coronary occlusion, which was successfully managed by electrical defibrillation.

Conventional anastomotic suturing was accomplished in $10 \pm 1$ minutes $^{16}$ and was accompanied by severe ventricular ectopic activity during coronary occlusion and on reperfusion, leading to ventricular fibrillation in one animal. 


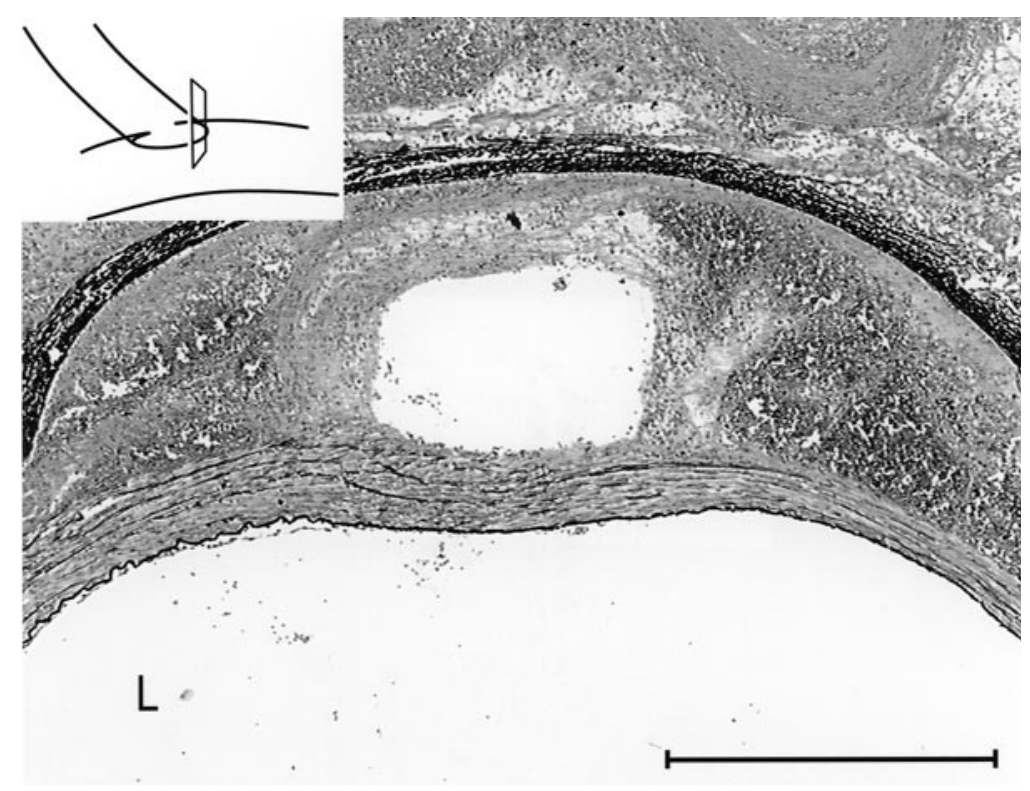

Fig 6. Transverse cross section of the toe of the anastomosis, 2 days after the operation. The blank space corresponds to the position of a clip that had penetrated and subsequently separated the adventitia from the media. The space between these 2 wall layers was plugged with platelet thrombi. (Elastin van Gieson stain; bar, $500 \mu \mathrm{m}$.) $L$, Lumen.

Graft blood flow and coronary hyperemic response, postoperative and at follow-up, are shown in Table I.

At follow-up, all anastomoses were patent angiographically (Fig 3). In 13 of 14 one-shot anastomoses, there was no evidence of luminal stenosis to be attributed to intravascular thrombus formation or excessive anastomotic intimal hyperplasia. In one anastomosis (28 days survival), however, the floor of the LAD opposite the anastomotic orifice appeared indented, and the angiographic stenosis was estimated to be $40 \%$.

Histologic analysis. In 9 of 14 one-shot anastomoses, the intima of the LAD was perfectly apposed to the intima of the ITA graft along its full circumference (Fig 4). In 5 cases, however, the LAD had not been adequately apposed at the heel $(\mathrm{n}=3)$ or lateral wall $(\mathrm{n}=$ 2) of the anastomosis (Fig 5). In the latter 2, anastomotic disruption was prevented by adventitial connective tissue between the clip tips. Exposed adventitia was covered by small platelet aggregates. In 3 cases, a clip had penetrated the adventitia at the toe of the anastomosis (Fig 6). In another 2 cases, the media and adventitia close to the anastomotic line had been separated entirely throughout the anastomosis (Fig 7, A), creating a false lumen distally over a length of 1 to 2 $\mathrm{mm}$ (Fig 7, B). In 8 anastomoses, 1 to 2 clips had penetrated the coronary arterial wall and were exposed to flowing blood. No mural thrombus formation, however, was observed on these clip ends or in the vicinity of the anastomoses. At both 2 and 28 days, we observed that the clips were slightly rotated inward in all anastomoses (Figs 7 and 8). The part of the circumference of the coronary arterial wall taken between the clip was maximally $1 \mathrm{~mm}$ in length.

After conventional anastomosis, intimal approximation was adequate circumferentially in all cases.

Endothelial injury. At 2 days, there were almost no endothelial cells left in the mid-segment of the anastomosis (Table II). Two millimeters downstream, however, the endothelial cell layer was nearly intact. Throughout the anastomotic segment, multiple small disruptions of the internal elastic lamina were observed (Fig 9), either alone or in combination with a medial tear. Endothelial cells were only scarcely noted at the everted distal end of the ITA graft. At 28 days, all anastomoses were completely re-endothelialized.

In the conventionally sutured anastomoses, endothelial damage was limited to only $5 \%$ to $10 \%$ of the circumference of the LAD and localized at the suture line. ${ }^{16}$

Medial necrosis. No medial necrosis was observed in the part of the vessel wall that was everted between 2 adjacent clips. Only the media that was included in the clip showed full thickness necrosis. The media close to the anastomotic line, and at the floor of the anastomosis opposite the arteriotomy, was generally infiltrated 

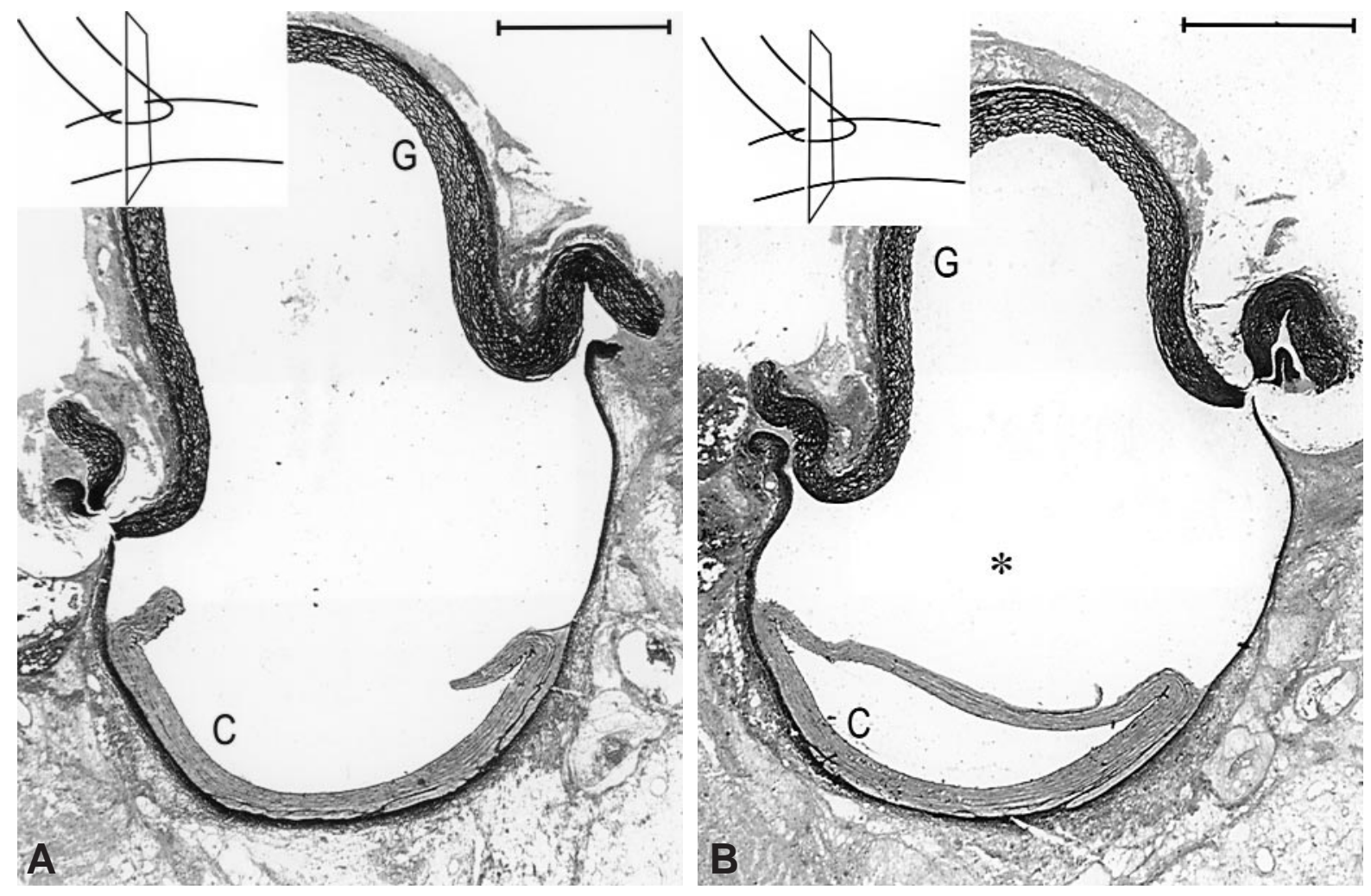

Fig 7. Transverse cross section of the anastomotic line in the mid-segment of the anastomosis, 2 days after the operation. In 2 of 14 anastomoses, the media and adventitia close to the anastomotic line had been separated entirely throughout the anastomosis (A), creating a false lumen $\left(^{*}\right)$ distally (B). (Elastin van Gieson stain; bar, $1000 \mu \mathrm{m}).(G$, Graft; $C$, coronary artery.

with polymorphonuclear lymphocytes without extensive necrosis.

Intimal hyperplasia. At 28 days, minimal intimal hyperplasia was seen at the heel, toe, and anastomotic lining. The anastomotic recess between the everted LAD and ITA graft was filled out with a small amount of neointima, $0.16 \pm 0.04 \mathrm{~mm}^{2}$ (Fig 8), although more pronounced when compared to 2 conventionally sutured anastomoses, 0.08 and $0.08 \mathrm{~mm}^{2} .{ }^{16}$

\section{Discussion}

The principal results of this study are the following: (1) In 8 of 14 anastomoses, all 12 clips were successfully applied in one shot. (2) Coronary artery occlusion was limited to approximately 3 minutes. (3) In spite of manifest early endothelial and medial damage and 2 local dissections, all anastomoses remained patent with minimal intimal hyperplasia at 4 weeks.

One-shot anastomotic stapler. In applying the oneshot device during ITA-LAD grafting on the beating heart, separate procedural steps can be identified, in- cluding exposure of the anastomotic area, eversion of the ITA graft, arteriotomy, and insertion and subsequent closure of the clips.

Exposure of the anastomotic area. The angled tip of the one-shot anastomotic stapler is intended for an angled end-to-side anastomosis. For adequate insertion of the tip, however, an angled approach is required, which is limited by both sternal edges and the lengthy firing handle. In addition, the present prototype required a recipient vessel of at least 2.5 to $3 \mathrm{~mm}$ in diameter. The proximal one third of the porcine LAD matched the required size but entailed extensive displacement of the beating heart to permit the necessary angled approach.

Eversion of the ITA graft. Before the distal part of the ITA graft was pulled through the clip cartridge, loose periadventitial tissue and hemostatic clips on side branches needed to be removed. Adventitial stripping, and hence disruption of vasa vasorum, however, may lead to mural ischemia and endothelial dysfunction, ${ }^{19}$ which may favor the occurrence of acute vasospasm. 
Table II. Percentage circumference of LAD not covered by endothelium

\begin{tabular}{lc}
\hline Location & $\begin{array}{c}\text { Endothelial denudation } \\
(\%)\end{array}$ \\
\hline$+2 \mathrm{~mm}$ & $6 \pm 3$ \\
Toe & $75 \pm 14$ \\
Mid & $92 \pm 5$ \\
Heel & $49 \pm 15$ \\
$-2 \mathrm{~mm}$ & $15 \pm 8$
\end{tabular}

Data are presented as mean \pm SEM.

Furthermore, later closure of side branches close to the anastomotic line by a hemostatic clip or suture can be demanding. In the present study, the ITA was harvested in the skeletonized fashion. A pedicled ITA graft, however, may be incompatible with the design of the current prototype.

The end of the graft was cut at an angle that conformed to the angled tip of the clip cartridge. Diversity in graft diameters encountered was matched by varying the graft angle.

Eversion of the distal end of the ITA is a delicate maneuver but could be performed in less than 1 minute. In most cases, however, the everted graft could not be secured on the lower jaws of the clips at the toe. Alternatively, the toe of the ITA was attached to a small pin proximal to the clips. On closure, the upper jaws of the clips had to grasp and subsequently evert the LAD, while being covered by the ITA. To enable adequate closure of the clips, we released the ITA graft from the pin on firing.

Arteriotomy. To prevent tearing of the arteriotomy edges on insertion of the everted ITA graft, the arteriotomy was created by means of an elliptic vascular punch. The use of a vascular punch, however, is to the detriment of the circumference of the recipient vessel. In addition, firing the punch was not a gentle maneuver and may inadvertently damage the intima.

Insertion and subsequent closure of the clips. Insertion of the everted ITA graft, together with close inspection of the arteriotomy edges, is a most delicate procedure. Because of the elasticity of the undersized arteriotomy in the normal porcine coronary artery, its edges tightly enclosed the everted distal end of the ITA after insertion. This ensured the adequate grip of the upper clip tips while closing, to enable circumferential eversion of the coronary arterial wall. Because of the mismatch in size, the one-shot device had to be inserted by means of a pivoting motion, while substantial pressure was exerted on the LAD. In spite of intimaintima contact while pivoting the one-shot device, there

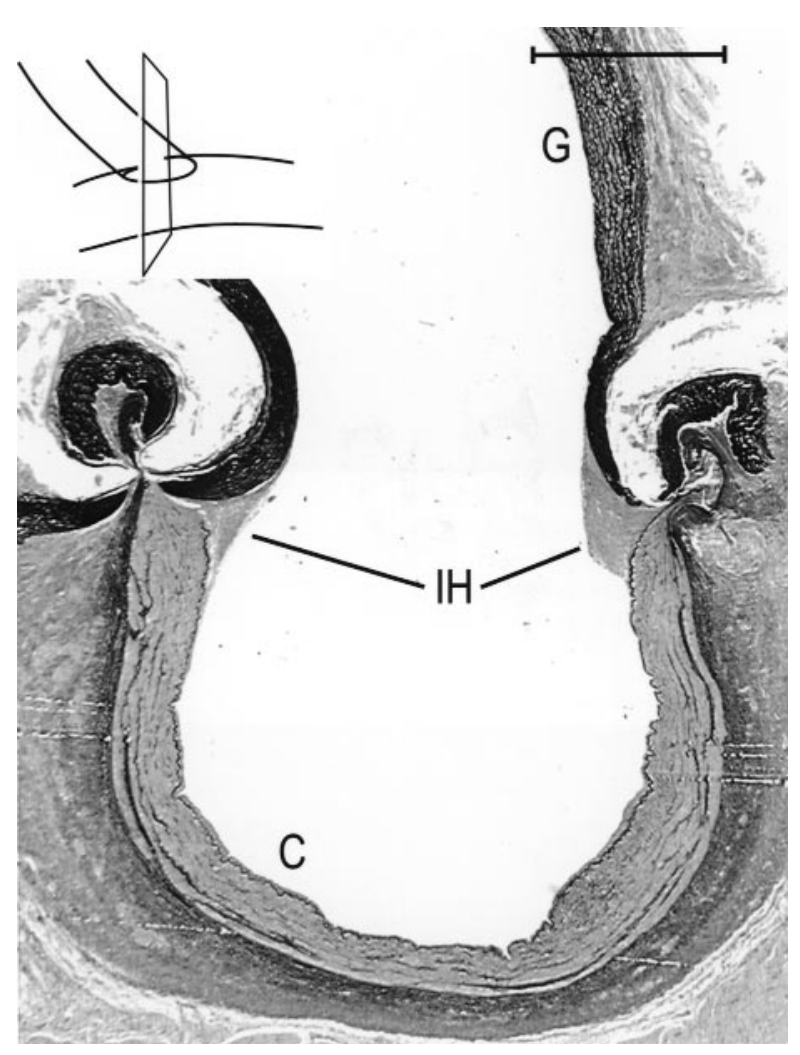

Fig 8. Transverse cross-section of the anastomotic line in the mid-segment of the anastomosis, 28 days after the operation. The anastomotic recess between the everted coronary artery and graft was completely filled out with neointima. (Elastin van Gieson stain; bar, $1000 \mu \mathrm{m}$.) $G$, graft; $I H$, intimal hyperplasia; $C$, coronary artery.

was a considerable loss of endothelial cells. Furthermore, in 2 anastomoses the media and adventitia close to the anastomotic line had separated, creating a false lumen distally. In neither of the 2 cases was the dissection noted intraoperatively, despite close inspection of the anastomotic line. To discriminate whether the everted arteriotomy edge consisted of all 3 vessel wall layers (Fig 4) or the adventitia only (Fig 7) was demanding. An incomplete vessel wall may constitute a risk of dissection. Because of the angled approach, the arteriotomy edge at the heel of the anastomosis could not be visualized satisfactorily. In 3 anastomoses, histologic evidence revealed inadequate eversion of the heel and hence imperfect apposition. It is conceivable that the heel-side of the cartridge was not properly inserted. Anastomotic disruption, however, was prevented by adventitial connective tissue of the coronary artery between the clip tips. In these respects, the design of the present prototype requires further improvements. 


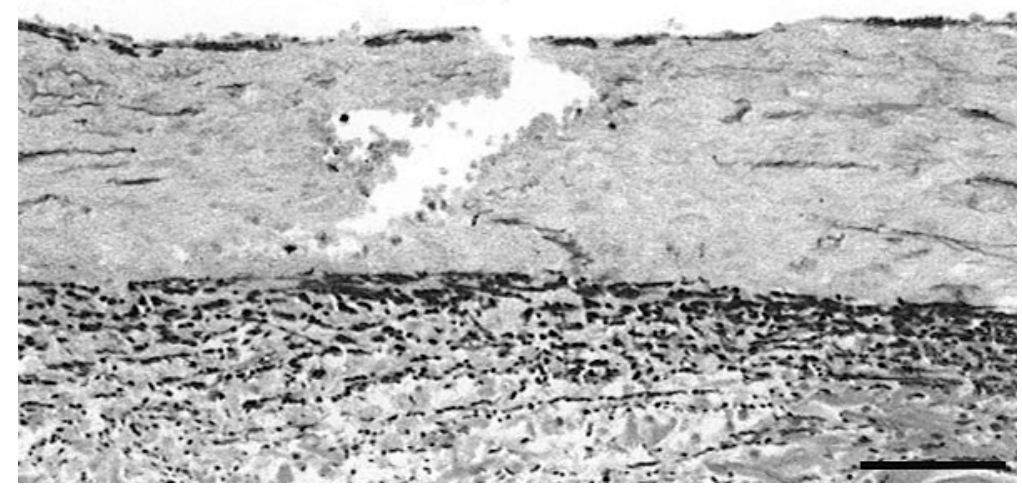

Fig 9. Transverse cross-section of the lateral wall of the LAD in the mid-segment of the anastomosis, 2 days after the operation. Throughout the anastomotic segments, multiple small disruptions of the intimal elastic lamina were observed, either alone or in combination with a medial tear. (Elastin van Gieson stain; bar, $100 \mu \mathrm{m}$.)

In spite of manifest early endothelial and medial damage, minimal intimal hyperplasia was seen at the heel, toe, and anastomotic lining at 28 days after the operation. In one anastomosis, angiography revealed a marked elevation of the floor of the LAD opposite the arteriotomy. Histologically, this could not be ascribed to mural thrombosis or excessive intimal hyperplasia.

Clinical application. In general, the skeletonized ITA will provide a sufficiently long, free segment for eversion of the distal end of the graft. Adequate eversion of arteriotomy edges, however, requires essentially a nonatherosclerotic and hence flexible, coronary arterial wall. ${ }^{15}$ In addition, a diseased coronary artery may be prone to dissection of the arterial wall on insertion. The present prototype needs to be downscaled to comply with the size of human internal thoracic arteries and coronary arteries in clinical practice.

\section{Conclusions}

The early prototype of the one-shot anastomotic stapler enabled short-occlusive, sutureless, end-to-side ITA-LAD grafting on the beating heart in the pig. In spite of early endothelial and medial damage and 2 local dissections, all anastomoses remained patent with minimal intimal hyperplasia at 4 weeks. Provided technical improvements are made, the device holds promise for clinical application. Its reliable functioning in atherosclerotic coronary arteries with variable wall thickness remains to be established. Ultimately, a modified version of this mechanical anastomotic device may facilitate the development of true porthole, closedchest, off-pump coronary operations.
We acknowledge the contributions of Jacqueline Bastiaanse, MD, Patrick Flanagan, Merel Schurink, Maringa Emons, and Hans W. G. Vosmeer and colleagues from the Utrecht University Central Animal Facilities.

\section{REFERENCES}

1. Benetti FJ, Naselli G, Wood M, Geffner L. Direct myocardial revascularization without cardiopulmonary bypass: experience in 700 patients. Chest 1991;100:312-6.

2. Moshkovitz Y, Lusky A, Mohr R. Coronary artery bypass without cardiopulmonary bypass: analysis of short-term and mid-term outcome in 220 patients. J Thorac Cardiovasc Surg 1995;110:979-87.

3. Buffolo E, de Andrade CS, Branco JN, Teles CA, Aguiar LF, Gomes WJ. Coronary artery bypass grafting without cardiopulmonary bypass. Ann Thorac Surg 1996;61:63-6.

4. Pfister AJ, Zaki MS, Garcia JM, Mispireta LA, Corso PJ, Qazi AG, et al. Coronary artery bypass without cardiopulmonary bypass. Ann Thorac Surg 1992;54:1085-92.

5. Benetti FJ, Mariani MA, Sani G, Boonstra PW, Grandjean JG, Giomarelli P, et al. Video-assisted minimally invasive coronary operations without cardiopulmonary bypass: a multicenter study. J Thorac Cardiovasc Surg 1996;112:1478-84.

6. Subramanian VA. Less invasive arterial CABG on a beating heart. Ann Thorac Surg 1997;63:S68-71.

7. Calafiore AM, DiGiammarco G, Teodori G, Bosco G, D'Annunzio E, Barsotti A, et al. Left anterior descending coronary artery grafting via left anterior small thoracotomy without cardiopulmonary bypass. Ann Thorac Surg 1996;61:1658-65.

8. Jansen EWL, Gründeman PF, Borst C, Eefting F, Diephuis J, Nierich A, et al. Less invasive off-pump CABG using a suction device for immobilization: the "Octopus" method. Eur J Cardiothorac Surg 1997;12:406-12.

9. Stevens JH, Burdon TA, Peters WS. Port-Access coronary artery bypass grafting: a proposed surgical method. J Thorac Cardiovasc Surg 1996;111:567-73.

10. Mack MJ, Acuff TE, Casimir-Ahn H, Lönn UJ, Jansen EWL. 
Video-assisted coronary bypass grafting on the beating heart. Ann Thorac Surg 1997;63:S100-3.

11. Werker PMN, Kon M. Review of facilitated approaches to vascular anastomosis surgery. Ann Thorac Surg 1997;63:S122-7.

12. Kirsch WM, Zhu YH, Hardesty RA, Chapolini R. A new method for microvascular anastomosis. Am Surg 1992;58:722-7.

13. Boeckx WD, Darius O, van den Hof B, van Holder C. Scanning electron microscopic analysis of the stapled microvascular anastomosis in the rabbit. Ann Thorac Surg 1997;63:S128-34.

14. Schild AF. Use of a vascular staple device for creation of AV fistulas and bridge grafts for hemodialysis. In: Henry ML, Ferguson RM, editors. Vascular access for hemodialysis-V. Chicago: Precept Press; 1997. p. 95-102.

15. Nataf P, Kirsch W, Hill AC, Anton T, Zhu YH, Ramadan R, et al. Nonpenetrating clips for coronary anastomosis. Ann Thorac Surg 1997;63:S135-7.
16. Heijmen RH, Borst C, van Dalen R, Verlaan CWJ, Mouës CM, van der Helm YJM, et al. Temporary luminal arteriotomy seal: II. Coronary artery bypass grafting on the beating heart. Ann Thorac Surg 1998;66:471-6.

17. Borst C, Jansen EWL, Tulleken CAF, Gründeman PF, Mansvelt Beck HJ, van Dongen JWF, et al. Coronary artery bypass grafting without cardiopulmonary bypass and without interruption of native coronary flow using a novel anastomosis site restraining device ("Octopus"). J Am Coll Cardiol 1996; 27:1356-64.

18. Heijmen RH, Gründeman PF, Borst C. Intima-adventitia apposition in end-to-side arterial anastomosis: an experimental study in the pig. Ann Thorac Surg 1998;65:705-11.

19. Margic K. Early changes in dissected small vessels: experimental study on rat arteries and veins. Plast Reconstr Surg 1985; 75:375-83.

\section{Online-www.aats.org}

Now you can get The Journal of Thoracic and Cardiovascular Surgery online. The Journal online brings you faster delivery time, easy searching of current and back issues, links to PubMed, AATS, WTSA and other important sites, and more. Visit the Journal online today. 